HIV in the UK and it is estimated that $15-20 \%$ of inmates at HMP Brixton are HIV positive.

Methods Staff delivered 26 walk-in clinics at a drug rehabilitation unit in Brixton over 5 months. The testing service was developed in close liaison with the drug and alcohol team and publicised using posters and education of reception staff. Oral swab testing for HIV and HCV was offered. A simple questionnaire was completed by attendees covering testing history and risk practice.

Results 35 individuals tested for HIV and HCV and were $80 \%$ male, $74.3 \%$ UK born, $48.6 \%$ white and $77.1 \%$ heterosexual. $37.1 \%$ were IVDU, median age was 36 (range 22-74). 57.1\% individuals had previously tested for HIV, median time since last test was 4 years (range $<1-15$ years). $54.3 \%$ individuals had previously tested for $\mathrm{HCV}$, median time since last test was 3 years (range $<1-11$ years). Median length of time in prison was 3 months (range $<1-48$ months). $48.6 \%$ and $51.4 \%$ had never been offered an HIV or HCV test in prison respectively. Only two individuals had tested for HIV and HCV in Prison. 14.3\% individuals were already known to be $\mathrm{HCV}$ positive. $5.7 \%(\mathrm{~N}=2)$ individuals were newly diagnosed $\mathrm{HCV}$ positive but none tested HIV positive. 82.9\% felt HIV/HCV testing should be offered in Prison. $71.1 \%$ felt this should be done using a mouth swab. 35\% had no concerns regarding HIV/HCV testing but in those who did, dislike of needles, receiving a positive result and concerns regarding confidentiality were the commonest barriers to testing.

Conclusions Prevalence of previously undiagnosed HCV was high in this group but HIV prevalence was zero. Uptake of HIV/HCV testing was very low despite good publicity and support from the drug services. This suggests that this method is not a cost effective approach to HIV testing despite oral swabs representing an acceptable mode of testing. Half of attendees had not been offered an HIV or HCV test in prison which needs to be increased. Testing in drug rehabilitation services does not result in high uptake of testing or detection of undiagnosed HIV.

\section{LBP-1.13 LEEP CONISATION AND THE RISK FOR PRETERM BIRTH: NEW HEALTH REGISTRY BASED DATA FROM FINLAND}

doi:10.1136/sextrans-2011-050119.19

1J Paavonen, ${ }^{1} \mathrm{~A}$ Heinonen, ${ }^{2} \mathrm{M}$ Gissler, ${ }^{1} \mathrm{~A} \mathrm{M}$ Tapper, ${ }^{1} \mathrm{M}$ Jakobsson. ${ }^{1} \mathrm{Helsinki}$ University Hospital, Helsinki, Finland; ${ }^{2}$ National Institute of Health and Welfare, Finland

Objectives To study whether the increasing severity of cervical intraepithelial neoplasia (CIN) by LEEP (loop electrosurgical excicion procedure) cone correlates with the risk for preterm birth. We also wanted to study whether the time period from LEEP or repeat LEEP correlates with the risk.

Methods Retrospective register-based study from Finland during 1997-2009. We collected data from the Hospital Discharge Register. We linked the data with data from the Finnish Medical Birth Register. The study population consisted of 20011 women who had LEEP during 1998-2009 and a subsequent delivery during 1997-2009. Controls were women with no previous LEEP $(\mathrm{n}=430975)$. The main outcome measure was preterm birth $(<37$ gestational weeks) rate.

Results The risk for preterm birth was increased after LEEP (OR 1.82, CI 1.62 to 2.03). In primiparous women this risk was slightly lower OR 1.61 (CI 1.42 to 1.83). Repeat LEEP was associated with almost threefold risk for preterm birth (OR 2.71, CI 1.98 to 3.69). Increasing severity of CIN did not correlate with the preterm birth rate. LEEP for carcinoma in situ or microinvasive cancer, however, increased the risk threefold (OR 3.25, CI 1.92 to 5.50). The risk was also increased for HPV-related non-CIN lesions (OR 2.55, CI 1.72 to 3.78). Time period since LEEP was not associated with the risk for preterm birth. Adjusting for maternal age, parity, socio-economic status, or marital status, or history of previous preterm birth did not change the results.

Conclusion The risk for preterm birth was increased after LEEP, but was not associated with the severity of CIN. Repeat LEEP had highest risk. Unnecessary LEEP should be avoided especially among young women.

\section{LBP-1.14 GENITAL WARTS: CANADIANS' PERCEPTION AND HEALTH RELATED BEHAVIOURS}

doi:10.1136/sextrans-2011-050119.20

${ }^{1} \mathrm{M}$ Steben, ${ }^{2} \mathrm{D}$ Labelle. ${ }^{1}$ Institut national de santé publique du Québec, Montréal, Canada; ${ }^{2}$ Graceway pharmaceuticals, Canada

Background A high level of anxiety is associated with a diagnosis of genital warts but health-related behaviours are less well known.

Objective To gauge the perceptions of Canadians towards genital warts and associated treatments.

Methods A survey supported by an unrestricted grant from Graceway Canada was conducted in February 2011 by Leger Marketing using their online panel (LegerWeb). It included 17 questions (2 multiple-choice, 15 4-point rating from strongly agree to strongly disagree]) relating to genital wart perception plus nine demographic questions.

Results The survey was completed by 1520 Canadian adults aged $18-75+$ years, of which $52 \%$ were female. Fifty-two per cent of respondents stated that they would monitor an unrecognised spot on their genitals and only seek medical assistance if it did not go away. Only $43 \%$ said they would stop having sex until the spots were gone. Concerns of being judged by friends/family were high (44\%). Regarding prevention, $32 \%$ of respondents believed that monogamy would protect against genital warts, and $25 \%$ believed they are not at risk if they use a condom. Treatment preference was in favour of a cream rather than an invasive treatment $(58 \%)$, particularly among younger $(67 \%)$ and male respondents $(63 \%)$. Over $60 \%$ would worry that genital warts could not be resolved. Women were less likely to believe that genital warts could be cured (13\% vs $23 \%$ ), and more likely to believe that they would recur (46\% vs 42\%) see Abstract LBP-1.14 Figure 1.

Conclusion Among Canadians, genital warts were associated with a fair degree of social stigma and worries regarding their resolution, and future recurrences. Non-invasive treatment options were preferred.

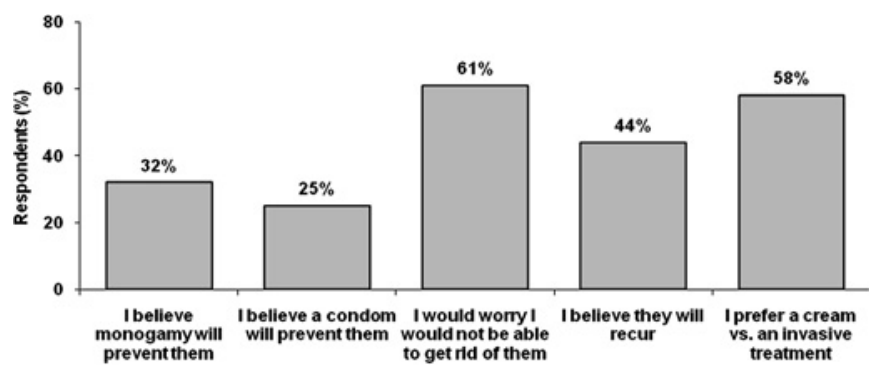

Abstract LBP-1.14 Figure 1

\section{LBP-1.15 AN EVALUATION OF THE LONG-TERM EFFECTIVENESS, IMMUNOGENICITY, AND SAFETY OF GARDASIL IN PREVIOUSLY VACCINATED WOMEN}

doi:10.1136/sextrans-2011-050119.21

A Saah. Merck Sharp \& Dohme, Whitehouse Station, USA

Background The GARDASIL long-term follow-up (LTFU) study is an ongoing extension of a pivotal randomised, placebo-controlled, 
double-blind, 4-year study to investigate the safety, immunogenicity, and effectiveness of quadrivalent Human Papillomavirus vaccine (qHPV) on the incidence of HPV 16/18-related cervical intraepithelial neoplasia (CIN) 2 or worse in 16-23-year-old women (Protocol 015).

Methods Follow-up of subjects will be accomplished in two ways: (1) registry-based follow-up for effectiveness data as well as safety data including but not limited to deaths, cancer, and hospitalisations; (2) active follow-up for blood collection for immunogenicity assessments at years 5 and 10 of the LTFU study. Effectiveness and safety analyses will occur approximately 2 years following completion of Protocol 015 and approximately every 2 years thereafter for 10 years. The current report represents the first of these efficacy and safety analyses. Cohort 1 included approximately 2700 subjects who received qHPV vaccine at the start of Protocol 015. Cohort 2 consists of approximately 2100 subjects who received placebo at the start of Protocol 015 and qHPV vaccine prior to entry into the LTFU. Vaccine effectiveness against
HPV 16/18-related CIN 2 or worse was estimated by calculating the expected incidence of CIN 2/3 or worse in an unvaccinated (placebo) cohort using historical registry data. The primary analysis approach was per-protocol.

Results There were 1080 subjects that contributed to the follow-up period out of a total of 2195 eligible subjects in the per-protocol population in Cohort 1. In these subjects there were no cases of HPV 16/18-related CIN 2 or worse observed. There were also no cases of HPV 6/11/16/18-related CIN, vulvar cancer, and vaginal cancer observed. However, the follow-up time in person-years is insufficient to make a definitive statement about the effectiveness of the qHPV vaccine for the current time period.

Conclusions The qHPV vaccine shows a trend of continued protection in women who were vaccinated up to 7 years previously, although there is as yet insufficient data to confirm that protection is maintained. The qHPV vaccine continues to be generally safe and well tolerated up to 6 years following vaccination. 